\title{
Axion Searches with the Storage Ring EDM Method
}

\author{
Seung Pyo Chang*, On Kim, Yannis K. Semertzidis \\ Department of Physics, KAIST, Daejeon 34141, Republic of Korea \\ Center for Axion and Precision Physics Research, IBS, Daejeon 34051, Republic of Korea \\ E-mail: koreanbrlo@gmail.com, bigstaron@kaist.ac.kr, \\ yannis@kaist.ac.kr
}

\section{Selcuk Haciomeroglu, Soohyung Lee, Seongtae Park}

Center for Axion and Precision Physics Research, IBS, Daejeon 34051, Republic of Korea

E-mail:Selcuk@ibs.re.kr, soohyunglee@ibs.re.kr, stpark@ibs.re.kr

\begin{abstract}
The axion-gluon coupling induces an oscillating electric dipole moment (EDM) in nucleons. The oscillating EDM can be detected using a storage ring EDM method with resonance between the $\mathrm{g}-2$ precession and the oscillating EDM frequencies. A frequency range from $\mathrm{mHz}$ to $100 \mathrm{MHz}$ can be scanned using the resonance method and one below $\mathrm{mHz}$ down to about $10^{-9} \mathrm{~Hz}$ can be searched parasitically with the frozen spin method. The estimated sensitivity is at the level of $10^{-30} \mathrm{e} \cdot \mathrm{cm}$ or higher. No experiment has accessed the proposed frequency range at such high sensitivity until now. In this study, we present the experimental method and compare the sensitivity with other experiments. (This work was supported by IBS-R017-D1-2018-a00.)
\end{abstract}

The 39th International Conference on High Energy Physics (ICHEP2018)

4-11 July, 2018

Seoul, Korea

${ }^{*}$ Speaker. 
The fact that the axion-gluon coupling can induce oscillating EDMs in nucleons [1] has led to a new idea of measuring the oscillating EDMs in hadronic particles such as protons and deuterons. We propose to use a storage ring technique to measure the axion induced oscillating EDM under resonance conditions between the axion frequency and the g- 2 spin precession frequency [2]. This method utilizes a combination of external electric and magnetic fields for resonance conditions to greatly improve the experimental sensitivity to the oscillating EDM.

In this study, we calculated the electric and magnetic fields required to create the resonance conditions for protons and deuterons. We also estimated achievable sensitivities in such conditions and found that the experiment is at least one order of magnitude more sensitive than the planned static EDM measurements, $\sim 10^{-30} \mathrm{e} \cdot \mathrm{cm}$. This higher sensitivity is achieved under the assumption that the axion frequency is priorly known and the experiment is set up for the frequency. At very low frequencies, $f_{\text {axion }} \leq 1 \mathrm{mHz}$, one can search for the axion with the sensitivities of $\sim 10^{-31} \mathrm{e} \cdot \mathrm{cm}$ using the frozen-spin method, without a prior knowledge of the axion frequency.

A wide range of frequencies $\left(10^{-9} \mathrm{~Hz} \sim 100 \mathrm{MHz}\right)$ of axion dark matter can be probed using both protons and deuterons in the same storage ring. Although the proposed method does not reach the sensitivity to the theoretical models of the QCD axion which could induce the oscillating EDMs, it promises to be one of the most sensitive ways to look for axions over a wide frequency range.
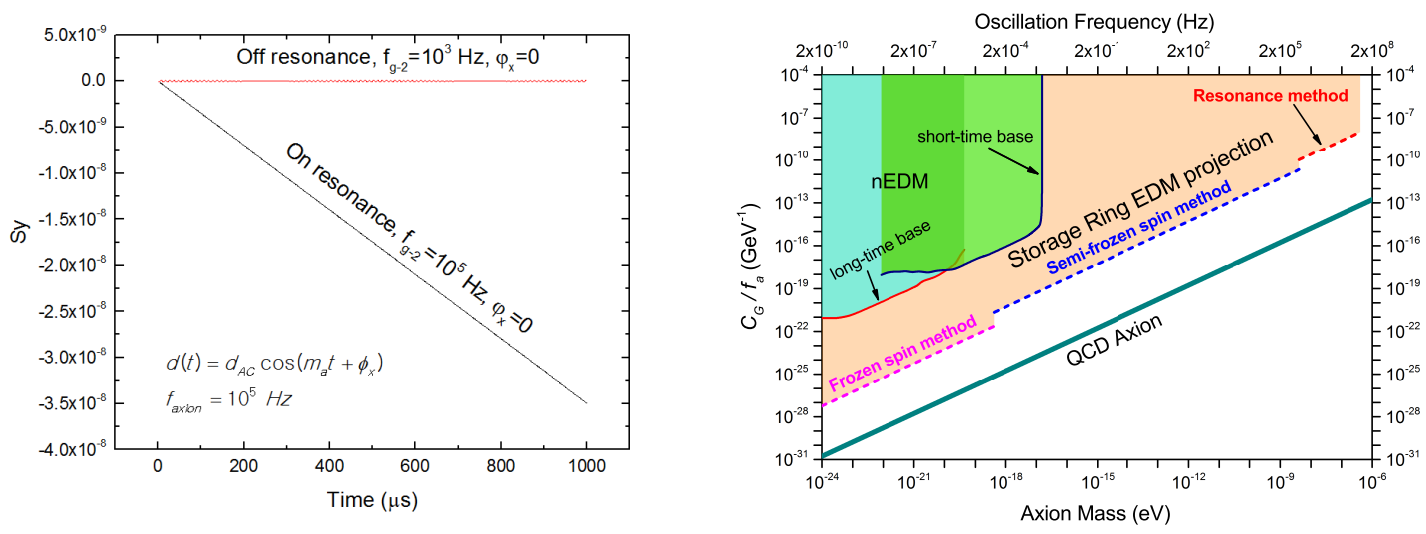

Figure 1: On- and off-resonance effects on the verti- Figure 2: Experimental limits for axion search using cal component of the spin of the source particles. the storage ring method.

\section{References}

[1] P. W. Graham and S. Rajendran, New observables for direct detection of axion dark matter, Phys. Rev. D 88 (2013) 035023.

[2] S. P. Chang, S. Haciomeroglu, O. Kim, S. Lee, S. Park and Y. K. Semertzidis, Axion dark matter search using the storage ring EDM method, PoS PSTP2017 (2018) 036 [1710.05271]. 